\title{
Efecto de la zona de mínimo oxígeno sobre la migración vertical de zooplancton gelatinoso en la bahía de Mejillones
}

\author{
Pedro Apablaza ${ }^{1} \&$ Sergio Palma ${ }^{1}$ \\ ${ }^{1}$ Escuela de Ciencias del Mar, Pontificia Universidad Católica de Valparaíso \\ Casilla 1020, Valparaíso, Chile
}

\begin{abstract}
RESUMEN. Se analizó la distribución estacional y migración vertical de zooplancton gelatinoso colectado en la bahía de Mejillones. Se identificaron 39 especies distribuidas en 14 de hidromedusas, 20 de sifonóforos y 5 de quetognatos. Las especies dominantes fueron Obelia spp., Liriope tetraphylla, Solmundella bitentaculata, Rophalonema velatum, Muggiaea atlantica, Sphaeronectes gracilis, Sagitta enflata y S. bierii, las que presentaron un estrecho rango de distribución vertical y migración nictimeral, debido a la influencia de la Zona de Mínimo Oxígeno (ZMO) $\left(<1 \mathrm{~mL} \cdot \mathrm{L}^{-1}\right)$, cuyo límite superior fue somero $(25-50 \mathrm{~m})$. En primavera la mayoría de las especies dominantes presentó desplazamientos nictimerales debido a la mayor oxigenación de la columna de agua. En cambio, en verano, debido a la termoclina más intensa y en invierno, cuando la ZMO se aproximó a la superficie, la mayoría de las especies dominantes no mostró cambios en su distribución vertical durante el ciclo diario. Obelia spp. tuvo una distribución somera, relacionada principalmente por la presencia de la termoclina. Mientras que $R$. velatum mostró una buena adaptación a las condiciones de escasez de oxígeno disuelto de la ZMO.
\end{abstract}

Palabras clave: distribución espacio-temporal, migración vertical, zona de mínimo oxígeno, bahía de Mejillones, Chile.

\section{The effect of the oxygen minimum zone on vertical migration of gelatinous zooplankton in Mejillones Bay}

\begin{abstract}
The seasonal distribution and vertical migration of gelatinous zooplankton collected in Mejillones Bay was analyzed. The 39 species identified included 14 hydromedusae, 20 siphonophores, and 5 chaetognates. The dominant species were Obelia spp., Liriope tetraphylla, Solmundella bitentaculata, Rophalonema velatum, Muggiaea atlantica, Sphaeronectes gracilis, Sagitta enflata, and S. bierii. Their vertical distribution and nictimeral migration ranges were narrow due to the influence of the Oxygen Minimum Zone $(\mathrm{OMZ})\left(<1 \mathrm{~mL} \cdot \mathrm{L}^{-1}\right)$, whose upper limit was quite shallow $(25-50 \mathrm{~m})$. In spring, the majority of the dominant species presented nictimeral movements due to the greater oxygenation of the water column. On the other hand, in summer, when the thermocline was more intense, and in winter, when the OMZ neared the surface, the majority of the dominant species showed no changes in their vertical distribution throughout the daily cycle. Obelia spp. had a shallow distribution, related principally to the presence of the thermocline. R. velatum, however, was well adapted to the scant dissolved oxygen in the OMZ.
\end{abstract}

Key words: spatial-temporal distribution, vertical migration, oxygen minimum zone, Mejillones Bay, Chile.

Autor corresponsal: spalma@ucv.cl

\section{INTRODUCCIÓN}

La península de Mejillones está inserta en el Sistema de la Corriente de Humboldt ( $\mathrm{SCH}$ ) y se caracteriza por frecuentes eventos de surgencia, donde afloran Aguas Ecuatoriales Subsuperficiales (AESS) asociadas a la corriente subsuperficial Perú-Chile, que generan la Zona de Mínimo Oxígeno (ZMO), con valores menores a $1 \mathrm{~mL} \cdot \mathrm{L}^{-1}$. Esta zona puede ser muy superficial en el norte de Chile y se pueden registrar bajas concentraciones de oxígeno disuelto a menos de $50 \mathrm{~m}$ de profundidad (Valdés \& Ortlieb, 2001). Las aguas superficiales de bajo contenido de oxígeno pueden ejercer una presión fisiológica sobre los organismos pelágicos y bentónicos, así como limitaciones a la migración vertical de los zooplancteres. En 
este sentido, se ha visto que algunas especies han desarrollado adaptaciones fisiológicas y/o de comportamientos especiales para subsistir en condiciones de mínimo oxígeno (Escribano \& Rodríguez, 1994; Escribano \& McLaren, 1999; Escribano et al., 2000; Santibáñez, 2003).

La bahía de Mejillones es una bahía abierta hacia el norte, con una profundidad máxima de $120 \mathrm{~m}$. Su ubicación la protege de las corrientes de dirección norte y favorece el desarrollo de un sistema de circulación interno que puede influir sobre las condiciones anóxicas del fondo (Valdés \& Ortlieb, 2001). Los bajos contenidos de oxígeno disuelto que caracterizan las aguas subsuperficiales de la bahía parecen haber permanecido durante los últimos tres milenios y se deberían principalmente, al ingreso constante de AESS por eventos de surgencia (Valdés \& Ortlieb, 2001), que favorecen la productividad de sus aguas, alcanzando uno de los valores más altos del norte de Chile (Marín et al., 1993).

En la bahía de Mejillones y áreas adyacentes, los estudios zooplanctónicos se han centrado principalmente en organismos quitinosos, como Calanus chilensis y Euphausia mucronata (Escribano \& Rodríguez, 1994, 1995; Marín, 1997; Escribano \& McLaren, 1999; Escribano \& Hidalgo, 2000; Escribano et al., 2000; Olivares, 2000; Santibáñez, 2003), siendo escasos aquellos referidos a gelatinosos (Pagès et al., 2001; Palma \& Apablaza, 2004).

En general, las especies gelatinosas habitan preferentemente las aguas neríticas y sus poblaciones declinan progresivamente hacia océanos abiertos, donde aparecen ejemplares aislados (Ulloa et al., 2000a). Estas especies suelen proliferar en zonas de surgencia costera de márgenes orientales, como $M$. atlantica, que es dominante en los sistemas de corrientes de Benguela (Pagès \& Gili, 1992) y Humboldt (Palma, 1994; Palma \& Rosales, 1995; Ulloa et al., 2000b; Palma \& Apablaza, 2004).

Como todos los seres marinos, el zooplancton habita un ambiente muy heterogéneo en tiempo y espacio, determinado por gradientes verticales físi$\cos$, químicos y factores biológicos. Estos gradientes están influenciados a macroescala por la circulación, traspaso de calor y estacionalidad; a mesoescala por surgencia y a microescala por mezcla vertical (Longhurst, 1976; Enright, 1977). En consecuencia, numerosas poblaciones planctónicas experimentan cambios en su distribución estacional y nictimeral (Palma, 1985; Pagès \& Gili, 1991; Andersen et al., 1992).
En el presente trabajo se analizarán las fluctuaciones estacionales de la ZMO en la bahía de Mejillones y los efectos que puede tener sobre la distribución y migración vertical de los organismos gelatinosos.

\section{MATERIALES Y MÉTODOS}

Entre el 17 de octubre de 2000 y el 19 de octubre de 2001, se efectuaron cuatro cruceros estacionales en la bahía de Mejillones $\left(23^{\circ} \mathrm{S}, 70,4^{\circ} \mathrm{W}\right)$ : $10-14$ octubre 2000 (primavera 2000), 10-14 febrero (verano), 10-14 agosto (invierno) y 10-14 octubre de 2001 (primavera 2001) (Fig. 1). En cada crucero se efectuaron pescas estratificadas de zooplancton durante un ciclo nictimeral de $24 \mathrm{~h}(10,14,18,22,02$ y 06 h), con redes WP-2 de $200 \mu \mathrm{m}$ de malla, provistas de flujómetros OSK para estimar el volumen filtrado. Las pescas se realizaron en los estratos de 0-25, 25-50 y 50-100 m de profundidad. Además, se efectuó un estrato adicional entre la superficie y la termoclina, determinado con un CTDO Seabird SBE-19 para analizar su efecto como barrera física a la migración nictimeral.

En cada ciclo diario se registraron los valores de temperatura, salinidad y contenido de oxígeno disuelto a intervalos de $8 \mathrm{~h}$, desde la superficie hasta cerca del fondo. Para describir las características oceanográficas de la columna de agua se utilizaron los perfiles verticales de CTDO obtenidos a las 10 $\mathrm{h}$ durante los cuatro periodos de muestreo. El límite superior de la ZMO se definió como la profundidad donde la concentración de oxígeno disuelto fue de 1 $\mathrm{mL} \cdot \mathrm{L}^{-1}$. Además, se calculó el porcentaje de participación de las masas de agua mediante el método del triángulo de mezcla (Mamayev, 1975).

Las muestras de zooplancton se preservaron con formalina al 5\% neutralizada con borato de sodio y de cada muestra se separaron e identificaron las medusas, sifonóforos y quetognatos. En sifonóforos, por su estructura colonial, se identificaron las fases poligástrica y eudoxia. El número de individuos se estandarizó y expresó en número de individuos por $1000 \mathrm{~m}^{3}$ de agua filtrada (ind $1000 \mathrm{~m}^{-3}$ ). Para el análisis de la migración nictimeral se consideraron solo las especies dominantes ( $\geq 5 \%$ del total) y la cantidad de organismos por estrato, se graficó como porcentaje del total colectado en la columna de agua en cada horario de muestreo.

Para separar los comportamientos nictimerales de las especies dominantes en los diferentes periodos 


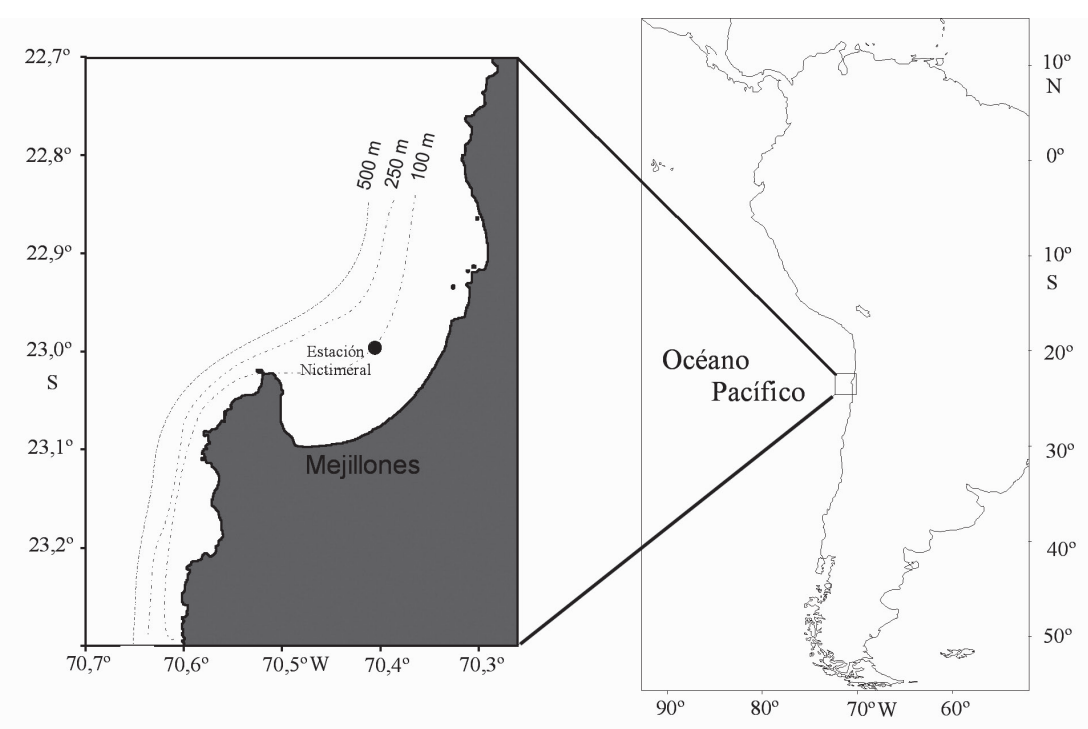

Figura 1. Estación de muestreo nictimeral en la bahía de Mejillones.

\section{Figure 1. Diel station in Mejillones Bay.}

estacionales, se realizó un análisis de función discriminante (Jongman et al., 1995), con el programa computacional Statistica 5.1. Previo a este análisis, se estableció una clasificación a priori de los comportamientos, considerando las diferencias observadas en la Profundidad Media Ponderada (PMP), calculada de la siguiente forma (Pillar et al., 1989):

$$
P M P=\frac{\sum n_{i} * d_{i}}{N}
$$

donde:

$\mathrm{d}_{\mathrm{i}}$ : profundidad media del estrato $\mathrm{i}$

$\mathrm{n}_{\mathrm{i}}$ : número de individuos en el estrato $\mathrm{i}$

$\mathrm{N}$ : total de individuos capturados en la columna de agua.

Como supuesto, se consideró que la migración nictimeral es cohesiva, es decir, que la mayoría de la población se mueve al mismo tiempo, con velocidad y profundidad inicial similar, lo que corresponde al modelo más general de migración planctónica (Pearre, 1979). En esta condición, se debería encontrar una sola moda en la distribución vertical de los individuos. De acuerdo a esto, se separaron las especies con comportamiento nictimeral normal, migración inversa y no migrante. Como migración normal se consideró aquella en que los organismos se encuentran a mayor profundidad en el día y más someros durante la noche.
Para analizar la relación entre las especies dominantes y las variables ambientales de temperatura, salinidad, oxígeno disuelto y densidad, la abundancia específica se normalizó con la transformación ln $(\mathrm{x}+1)$ y se utilizó el análisis de correlación de rangos de Spearman. En el caso de las variables ambientales se empleó el promedio ponderado por estrato de profundidad (Palma \& Apablaza, 2004).

\section{RESULTADOS}

\section{Condiciones oceanográficas}

En la bahía de Mejillones se determinó una estructura vertical de dos capas, una superficial bien oxigenada, con marcados gradientes verticales de temperatura y una subsuperficial más homogénea, con menores temperaturas y valores de oxígeno disuelto inferiores a $1 \mathrm{~mL} \cdot \mathrm{L}^{-1}$. El espesor de cada una de estas capas fue muy variable entre los periodos estudiados y fue determinado por la profundidad del límite inferior de la temoclina y la ubicación del límite superior de la ZMO.

En octubre de 2000 la distribución vertical de la temperatura y oxígeno disuelto permitieron detectar una capa superficial entre los 0 a $35 \mathrm{~m}$ de profundidad. La temperatura de esta capa varió entre 12,5 y $16^{\circ} \mathrm{C}$, la salinidad presentó un mínimo de 34,4 psu y alto contenido de oxígeno disuelto $\left(\sim 6 \mathrm{~mL} \cdot \mathrm{L}^{-1}\right)$ en los 
primeros $15 \mathrm{~m}$. Bajo la capa superficial, los valores de temperatura y oxígeno disuelto disminuyeron paulatinamente hasta alcanzar, valores mínimos cercanos a $12^{\circ} \mathrm{C}$ y $0,05 \mathrm{~mL} \cdot \mathrm{L}^{-1}$ respectivamente, a 100 $\mathrm{m}$ de profundidad. La salinidad en cambio, aumentó levemente a valores cercanos a 34,9 psu cerca del fondo (Fig. 2a).

Durante el muestreo realizado en febrero de 2001, el límite superior de la ZMO y la base de la termoclina se ubicaron a una profundidad de $45 \mathrm{~m}$, definiendo una capa superficial con temperaturas mínimas cercanas a $13^{\circ} \mathrm{C}$ y máximas mayores a $17^{\circ} \mathrm{C}$. Al igual que en octubre del 2000 , los altos valores de oxígeno disuelto $\left(\sim 6 \mathrm{~mL} \cdot \mathrm{L}^{-1}\right)$, fueron detectados en los primeros $10 \mathrm{~m}$ de profundidad. $\mathrm{La}$ capa subsuperficial presentó una escasa variación de temperatura $\left(<0,5^{\circ} \mathrm{C}\right.$ entre los 45 y $\left.100 \mathrm{~m}\right)$ y un mínimo de oxígeno disuelto a $0,50 \mathrm{~mL} \cdot \mathrm{L}^{-1}$ cerca del fondo. La salinidad presentó una escasa variación de sus valores en toda la columna de agua, con mínimos de 34,4 psu en superficie y máximos de 34,6 psu a $100 \mathrm{~m}$ (Fig. 2b).

En agosto de 2001, el espesor de la capa superficial disminuyó considerablemente con respecto a los periodos anteriores, ubicándose a los $18 \mathrm{~m}$. Dentro de esta capa, la temperatura se mantuvo cercana a $14^{\circ} \mathrm{C}$ y el oxígeno disuelto alcanzó un máximo de $4 \mathrm{~mL} \cdot \mathrm{L}^{-1}$ en superficie. En la capa subsuperficial la temperatura se mantuvo sobre $\operatorname{los} 13^{\circ} \mathrm{C}$, mientras que el oxígeno disuelto disminuyó hasta valores cercanos a $0,03 \mathrm{~mL} \cdot \mathrm{L}^{-1}$ a partir de los $40 \mathrm{~m}$ de profundidad. La salinidad, al igual que en febrero, no presentó mayores variaciones verticales en la columna de agua y se mantuvo entre 34,7 y 34,9 psu (Fig. 2c).

En octubre de 2001, en la capa superficial (0-50 m) la temperatura fluctuó entre 13 y $16^{\circ} \mathrm{C}$; la salinidad presentó una estructura muy irregular, con un mínimo de 34,7 psu centrado a 20 m; y los máximos de oxígeno alcanzaron valores cercanos a $5,5 \mathrm{~mL} \cdot \mathrm{L}^{-}$ 1. La capa subsuperficial se mantuvo con valores próximos $13^{\circ} \mathrm{C}$, con mínimos de oxígeno disuelto $(<$ $0,5 \mathrm{~mL} \cdot \mathrm{L}^{-1}$ ) bajo los $80 \mathrm{~m}$ y salinidades entre $34,9 \mathrm{y}$ 35,0 psu (Fig. 2d).

En la bahía se determinó la presencia de tres masas de agua, Agua Subtropical (AST), Agua Subantártica (ASAA) y Agua Ecuatorial Subsuperficial (AESS), con mayor preponderancia de esta última en ambas primaveras e invierno. La capa superficial se caracterizó por la mezcla de AST y ASAA, con bajos porcentajes de AESS que aumentaron progresivamente en profundidad, alcanzando porcentajes superiores al $70 \%$ bajo los $30 \mathrm{~m}$. A profundidades mayores de $60 \mathrm{~m}$ se determinó la mezcla de ASAA y AESS, con un elevado porcentaje de esta última (Fig. 3).

\section{Composición taxonómica y abundancia estacional}

Durante el periodo de estudio se identificaron 39 especies de zooplancton gelatinoso, correspondientes a 14 hidromedusas, 20 sifonóforos y 5 quetognatos. En este periodo se determinaron las siguientes especies dominantes: Clytia spp., Liriope tetraphylla, Obelia spp., Rophalonema velatum, Solmundella bitentaculata, Muggiaea atlantica, Sphaeronectes gracilis, Sagitta bierii y S. enflata (Tabla 1).

La distribución estacional mostró máximos de abundancia en octubre y febrero (primavera-verano) y mínimos en agosto (invierno), patrón que fue seguido por la mayoría de las especies dominantes. En primavera se produjo un fuerte incremento en la cantidad de eudoxias de $M$. atlantica y $S$. gracilis (Tabla 1), así como de individuos juveniles de $L$. tetraphylla, Obelia spp., Clytia spp. y S. bitentaculata. Al comparar ambas primaveras, se observó que en octubre de 2001 la abundancia de gelatinosos fue mucho mayor que en octubre de 2000 (Tabla 1).

Las mayores agregaciones de medusas se registraron en primavera y verano, constituidas por altas densidades de Aglaura hemistoma, Clytia spp., Liriope tetraphylla, Obelia spp., Phialella quadrata, $R$. velatum y $S$. bitentaculata. En la primavera de 2000 ocurrieron los máximos de Clytia spp., mientras que los de A. hemistoma, Obelia spp., Phialella quadrata y $S$. bitentaculata ocurrieron en la primavera de 2001 (Tabla 1). En verano, la única medusa que presentó máximos de abundancia fue $L$. tetraphylla. En invierno, a excepción de Obelia spp. y L. tetraphylla, las medusas fueron muy escasas y su abundancia disminuyó en un orden de magnitud respecto al verano (Tabla 1).

Los sifonóforos Muggiaea atlantica y Sphaeronectes gracilis fueron abundantes en todos los periodos estacionales, inclusive en invierno (Tabla 1). Los máximos de $M$. atlantica se registraron en primavera y los de $S$. gracilis en verano. En invierno se registró un fuerte descenso tanto en abundancia como en número de especies, de hecho se obtuvo un $25 \%$ de las especies colectadas en verano. A excepción de $M$. atlantica y $S$. gracilis que constituyeron casi el $90 \%$ de los sifonóforos, las especies restantes fueron escasas u ocasionales. 



Oxİgeno disuelto $\left(\mathrm{mL} \cdot \mathrm{L}^{-1}\right)$
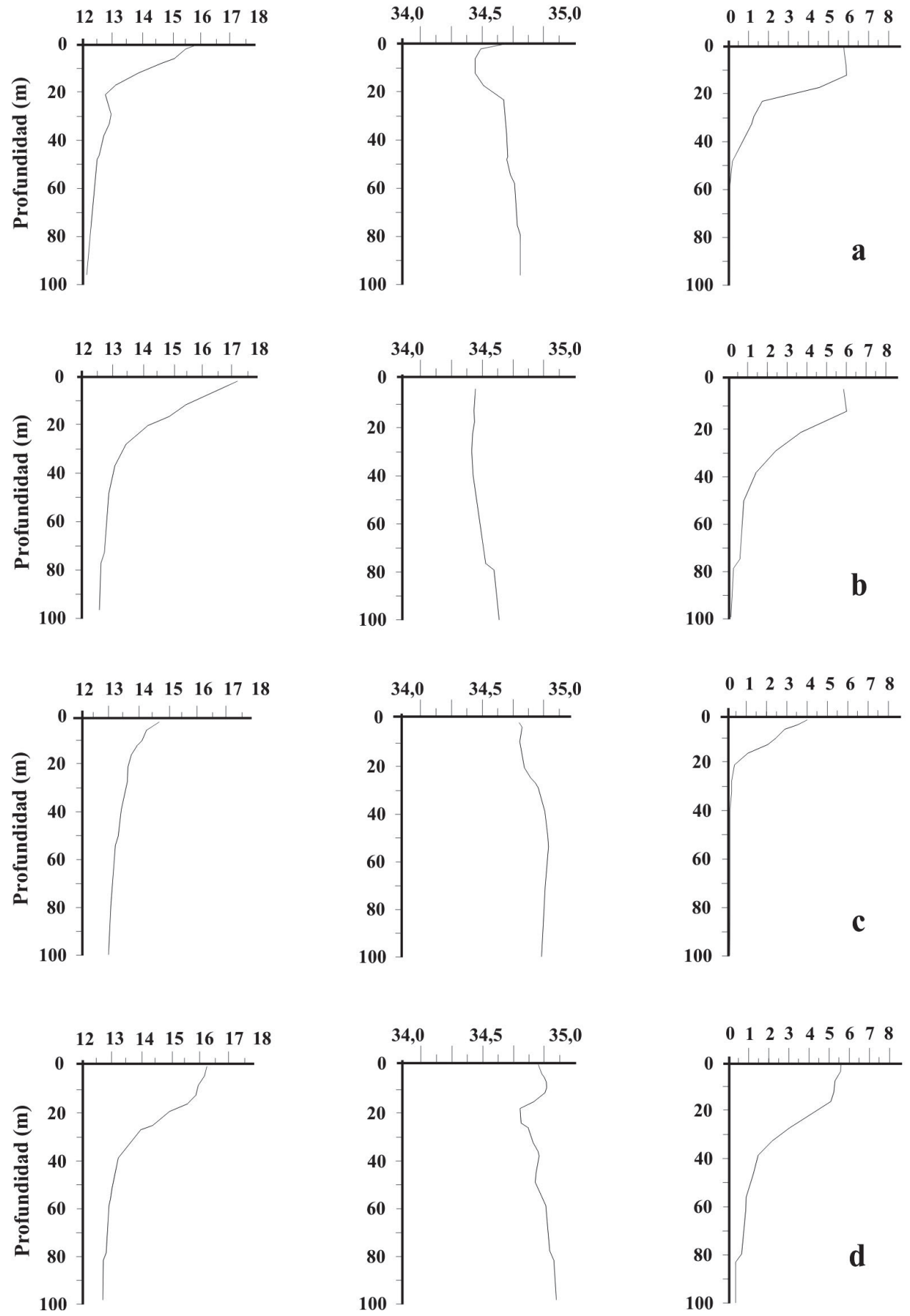

Figura 2. Distribución vertical de temperatura, salinidad y oxígeno disuelto. a) octubre 2000, b) febrero 2001, c) agosto 2001, y d) octubre 2001.

Figure 2. Vertical distribution of temperature, salinity and dissolved oxygen. a) October 2000, b) February 2001, c) August 2001, and d) October 2001. 
Tabla 1. Abundancia estacional media de los carnívoros gelatinosos colectados en la bahía de Mejillones (octubre 2000 - octubre 2001). DS: desviación estándar, C: colonia, FE: fase eudoxia, FP: fase poligástrica.

Table 1. Mean seasonal abundance of gelatinous carnivorous collected in Mejillones bay (October 2000 - October 2001). DS: standard deviation, C: colonies, FP: polygastric phase, FE: eudoxid phase.

\begin{tabular}{|c|c|c|c|c|c|c|c|c|}
\hline \multirow[t]{2}{*}{ Especie } & \multicolumn{2}{|c|}{$\begin{array}{c}\text { Octubre } \\
2000\end{array}$} & \multicolumn{2}{|c|}{$\begin{array}{c}\text { Febrero } \\
2001\end{array}$} & \multicolumn{2}{|l|}{$\begin{array}{c}\text { Agosto } \\
2001\end{array}$} & \multicolumn{2}{|c|}{$\begin{array}{c}\text { Octubre } \\
2001\end{array}$} \\
\hline & ind $\cdot 1000 \mathrm{~m}^{-3}$ & DS & ind $\cdot 1000 \mathrm{~m}^{-3}$ & DS & ind $\cdot 1000 \mathrm{~m}^{-3}$ & DS & ind $\cdot 1000 \mathrm{~m}^{-3}$ & DS \\
\hline \multicolumn{9}{|l|}{ Hydromedusae } \\
\hline Aglaura hemistoma & 1334 & 122 & 2433 & 140 & 42 & 9 & 8517 & 272 \\
\hline Amphogona alicata & 54 & 9 & 73 & 6 & 25 & 5 & 125 & 10 \\
\hline Clytia spp. & 5985 & 436 & 113 & 10 & 43 & 9 & 968 & 69 \\
\hline Cunina peregrina & 29 & 7 & 1321 & 86 & 164 & 15 & 937 & 35 \\
\hline Dipurena sp. & 0 & 0 & 6 & 1 & 0 & 0 & 0 & 0 \\
\hline Ectopleura dumortieri & 56 & 10 & 21 & 4 & 0 & 0 & 276 & 24 \\
\hline Euphysa aurata & 26 & 7 & 0 & 0 & 0 & 0 & 0 & 0 \\
\hline Liriope tetraphylla & 92 & 17 & 1816 & 61 & 709 & 35 & 759 & 44 \\
\hline Obelia spp. & 3051 & 234 & 4018 & 294 & 1067 & 63 & 11381 & 815 \\
\hline Phialella quadrata & 227 & 36 & 154 & 11 & 90 & 8 & 2365 & 194 \\
\hline Rhopalonema velatum & 2385 & 265 & 1106 & 60 & 225 & 22 & 1827 & 61 \\
\hline Sarsia eximia & 142 & 17 & 43 & 6 & 88 & 9 & 597 & 60 \\
\hline Solmaris rhodoloma & 1235 & 172 & 635 & 84 & 121 & 10 & 0 & 0 \\
\hline Solmundella bitentaculata & 13932 & 1487 & 1648 & 126 & 232 & 25 & 20144 & 934 \\
\hline \multicolumn{9}{|l|}{ Siphonophora } \\
\hline Abylopsis tetragona $(\mathrm{FP})$ & 54 & 16 & 957 & 78 & 0 & 0 & 446 & 27 \\
\hline Abylopsis tetragona (FE) & 0 & 0 & 1134 & 59 & 0 & 0 & 799 & 27 \\
\hline Agalma elegans & 83 & 21 & 274 & 24 & 15 & 3 & 372 & 30 \\
\hline Agalma elegans $(\mathrm{C})$ & 0 & 0 & 1229 & 84 & 332 & 29 & 97 & 7 \\
\hline Amphicaryon acaule & 0 & 0 & 85 & 10 & 0 & 0 & 243 & 23 \\
\hline Bassia bassensis (FP) & 27 & 7 & 1042 & 56 & 9 & 2 & 17 & 4 \\
\hline Bassia bassensis (FE) & 0 & 0 & 3671 & 172 & 16 & 2 & 372 & 33 \\
\hline Diphyes bojani (FE) & 0 & 0 & 62 & 9 & 0 & 0 & 0 & 0 \\
\hline Enneagonum hyalinum (FP) & 0 & 0 & 140 & 10 & 0 & 0 & 0 & 0 \\
\hline Enneagonum hyalinum (FE) & 0 & 0 & 105 & 10 & 0 & 0 & 13 & 3 \\
\hline Eudoxoides spiralis & 0 & 0 & 40 & 4 & 0 & 0 & 46 & 7 \\
\hline Hippopodius hippopus & 0 & 0 & 12 & 2 & 0 & 0 & 0 & 0 \\
\hline Lensia hardy & 0 & 0 & 26 & 3 & 0 & 0 & 0 & 0 \\
\hline Lensia hotspur & 45 & 8 & 81 & 6 & 0 & 0 & 70 & 6 \\
\hline Lensia challengeri & 0 & 0 & 122 & 13 & 0 & 0 & 285 & 15 \\
\hline Lensia fowleri (FE) & 0 & 0 & 5 & 1 & 0 & 0 & 0 & 0 \\
\hline Lensia subtilis & 0 & 0 & 5 & 1 & 0 & 0 & 0 & 0 \\
\hline Muggiaea atlantica & 8221 & 551 & 5382 & 253 & 3419 & 194 & 34274 & 1010 \\
\hline Muggiaea atlantica $(\mathrm{FE})$ & 0 & 0 & 4530 & 343 & 7985 & 425 & 49400 & 2217 \\
\hline Rhizophysa eysenhardti & 0 & 0 & 12 & 3 & 0 & 0 & 0 & 0 \\
\hline Rosacea alicata & 0 & 0 & 20 & 3 & 0 & 0 & 509 & 43 \\
\hline Sphaeronectes fragilis & 0 & 0 & 9 & 2 & 0 & 0 & 15 & 3 \\
\hline Sphaeronectes gamulini (FP) & 211 & 25 & 9 & 2 & 0 & 0 & 0 & 0 \\
\hline Sphaeronectes gracilis (FP) & 1667 & 130 & 2849 & 100 & 1017 & 48 & 2475 & 160 \\
\hline Sphaeronectes gracilis (FE) & 0 & 0 & 911 & 46 & 374 & 23 & 6555 & 448 \\
\hline Sphaeronectes irregularis (FP) & 0 & 0 & 1237 & 64 & 42 & 5 & 280 & 18 \\
\hline Sphaeronectes irregularis (FE) & 0 & 0 & 0 & 0 & 29 & 4 & 160 & 15 \\
\hline Fase poligástrica sp. & 0 & 0 & 401 & 26 & 0 & 0 & 15 & 3 \\
\hline Fase eudoxia sp. & 0 & 0 & 625 & 41 & 0 & 0 & 36 & 5 \\
\hline \multicolumn{9}{|l|}{ Chaetognatha } \\
\hline Krohnitta subtilis & 26 & 7 & 47 & 6 & 0 & 0 & 25 & 4 \\
\hline Sagitta virrey & 7098 & 394 & 30431 & 977 & 544 & 655 & 12194 & 389 \\
\hline Sagitta enflata & 53626 & 3720 & 59736 & 2127 & 1555 & 720 & 57912 & 1857 \\
\hline Sagitta minima & 114 & 20 & 399 & 42 & 0 & 0 & 637 & 46 \\
\hline Sagitta pacifica & 0 & 0 & 38 & 5 & 0 & 0 & 506 & 67 \\
\hline
\end{tabular}


Al igual que las dos especies dominantes de sifonóforos, los quetognatos Sagitta enflata y S. bierii fueron abundantes en todos los periodos analizados, con máximos en primavera y verano (Tabla 1). En invierno, al igual que en medusas, se observó una fuerte disminución en número de individuos y de especies, salvo $S$. bierii y $S$. enflata que estuvieron bien representadas (Tabla 1).

\section{Distribución vertical y migración nictimeral}

La mayoría de las especies dominantes, Obelia spp., L. tetraphylla, S. bitentaculata, M. atlantica y $S$. gracilis, se distribuyó en los primeros $50 \mathrm{~m}$, preferentemente sobre la ZMO (Figs. 4 y 5a). De ellas, Obelia spp. fue la más superficial $(0-25 \mathrm{~m})$ y no incursionó en la ZMO (Fig. 4a). El análisis de
Spearman indicó que la abundancia de estas especies tuvo una correlación positiva $(\mathrm{p}<0,05)$ con la temperatura y oxígeno disuelto, y negativa con la salinidad y densidad (Tabla 2).

El resto de las especies dominantes, $R$. velatum, $S$. enflata y $S$. bierii, mostró una distribución vertical más extensa, que abarcó toda la columna de agua (Figs. 5b, 5c y 5d). R. velatum se colectó principalmente entre 25 y $100 \mathrm{~m}$, a excepción de agosto que se distribuyó en los primeros 50 m; más del $70 \%$ de los ejemplares de esta especie se encontró bajo el límite superior de la ZMO (Fig. 5b) y tuvo una correlación negativa con la temperatura y oxígeno disuelto, y positiva con la densidad (Tabla 2). $S$. enflata se encontró más superficialmente en octubre de 2000 y agosto $2001(0-30 \mathrm{~m})$, mientras que en
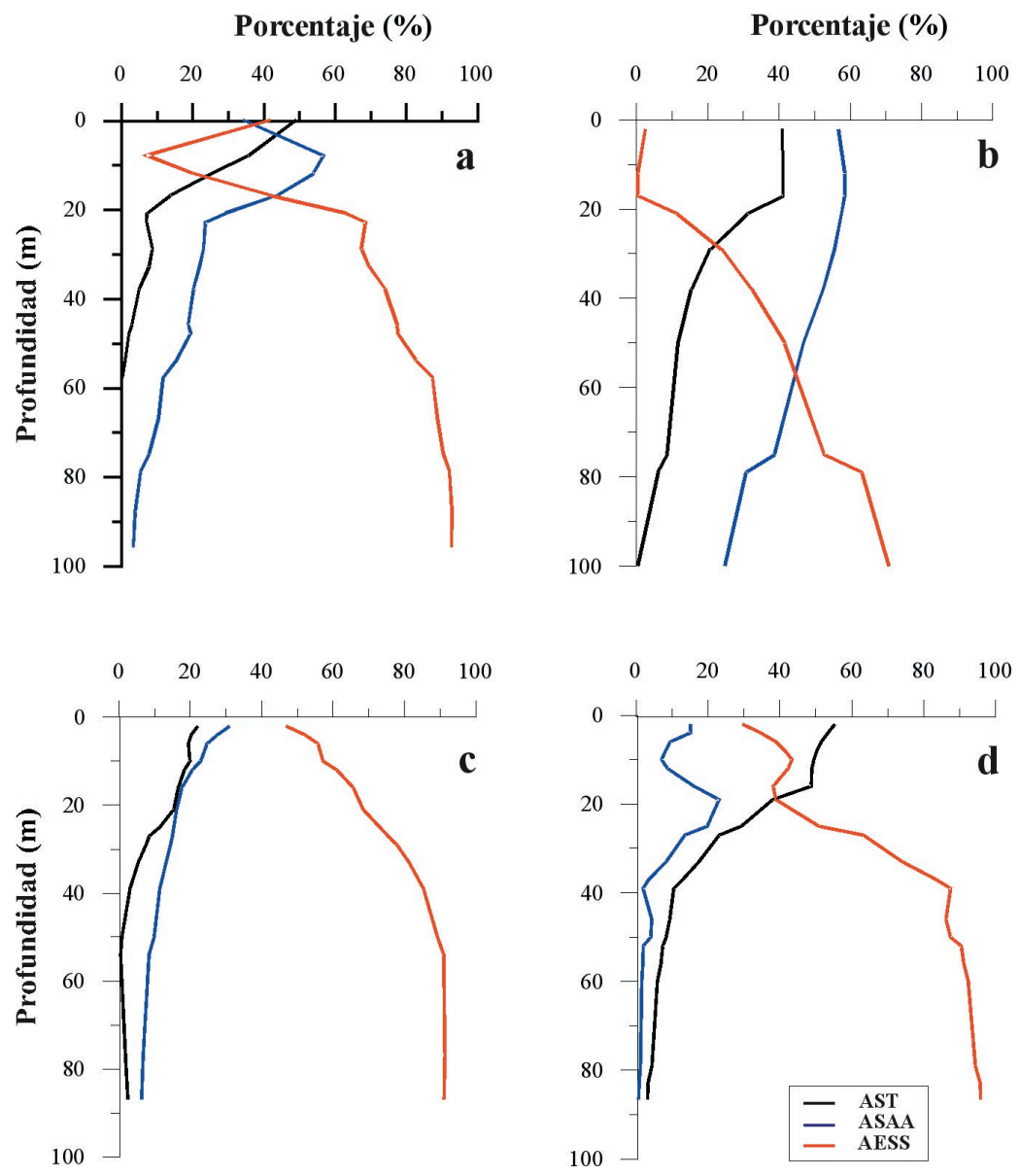

Figura 3. Distribución porcentual de las masas de agua en la estación nictimeral. AST: Agua Subtropical, ASAA, Agua Subantártica, AESS: Agua Ecuatorial Subsuperficial.

Figure 3. Percentage distribution of water masses in the diel station. AST: Subtropical Water, ASAA: Subantarctic Water, AESS: Equatorial Subsurface Water. 


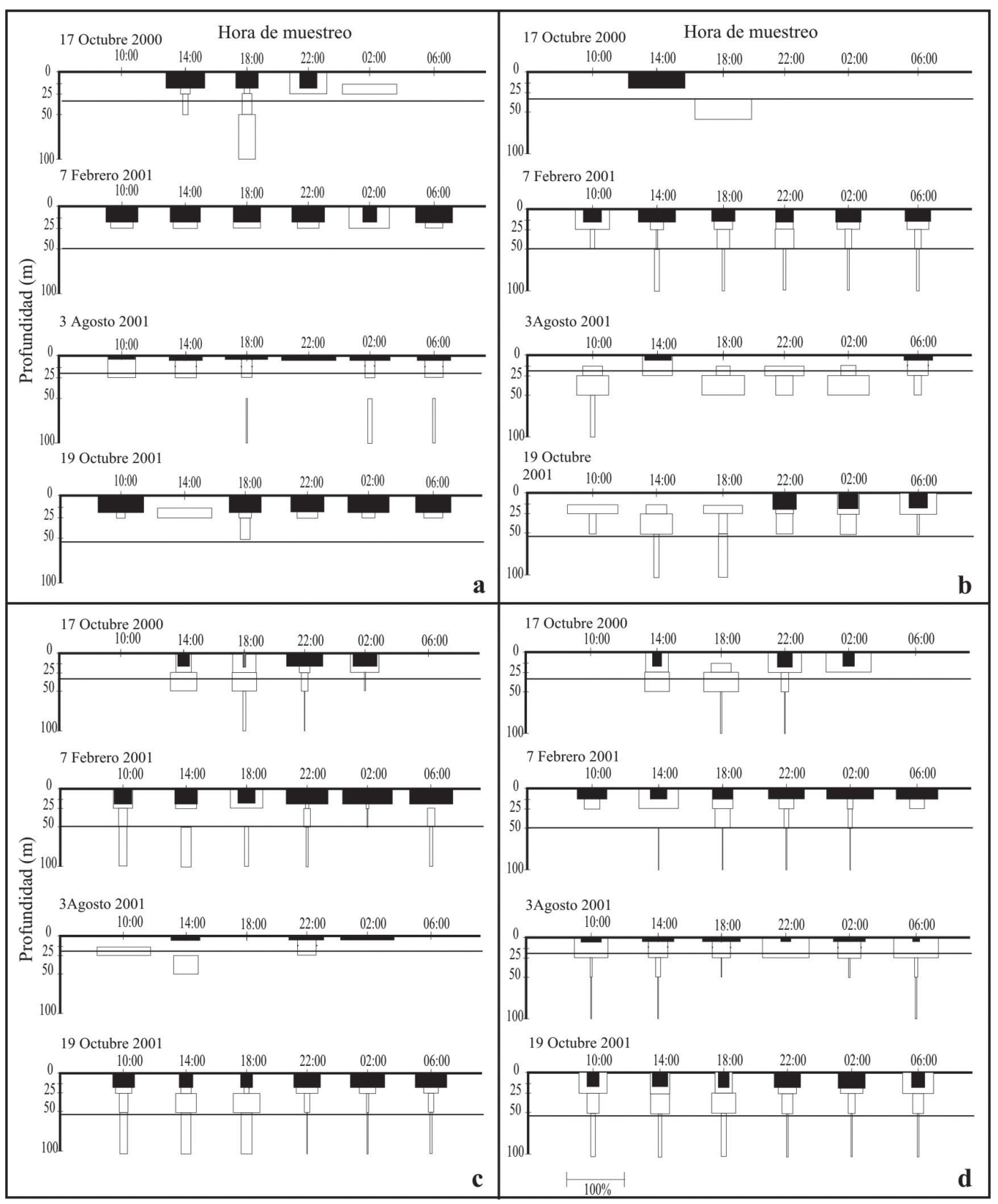

Figura 4. Distribución nictimeral de a) Obelia spp., b) Liriope tetraphylla, c) Solmundella bitentaculata y d) Muggiaea atlantica, en los distintos periodos de muestreo. La barra negra indica el porcentaje de individuos colectados sobre la termoclina y la línea negra el límite superior de la Zona de Mínimo Oxígeno.

Figure 4. Diel distribution of a) Obelia spp., b) Liriope tetraphylla, c) Solmundella bitentaculata and d) Muggiaea atlantica, in different sampling periods. The black bar corresponds to individuals collected above the thermocline. The black line indicates the upper limit of Oxygen Minimum Zone. 


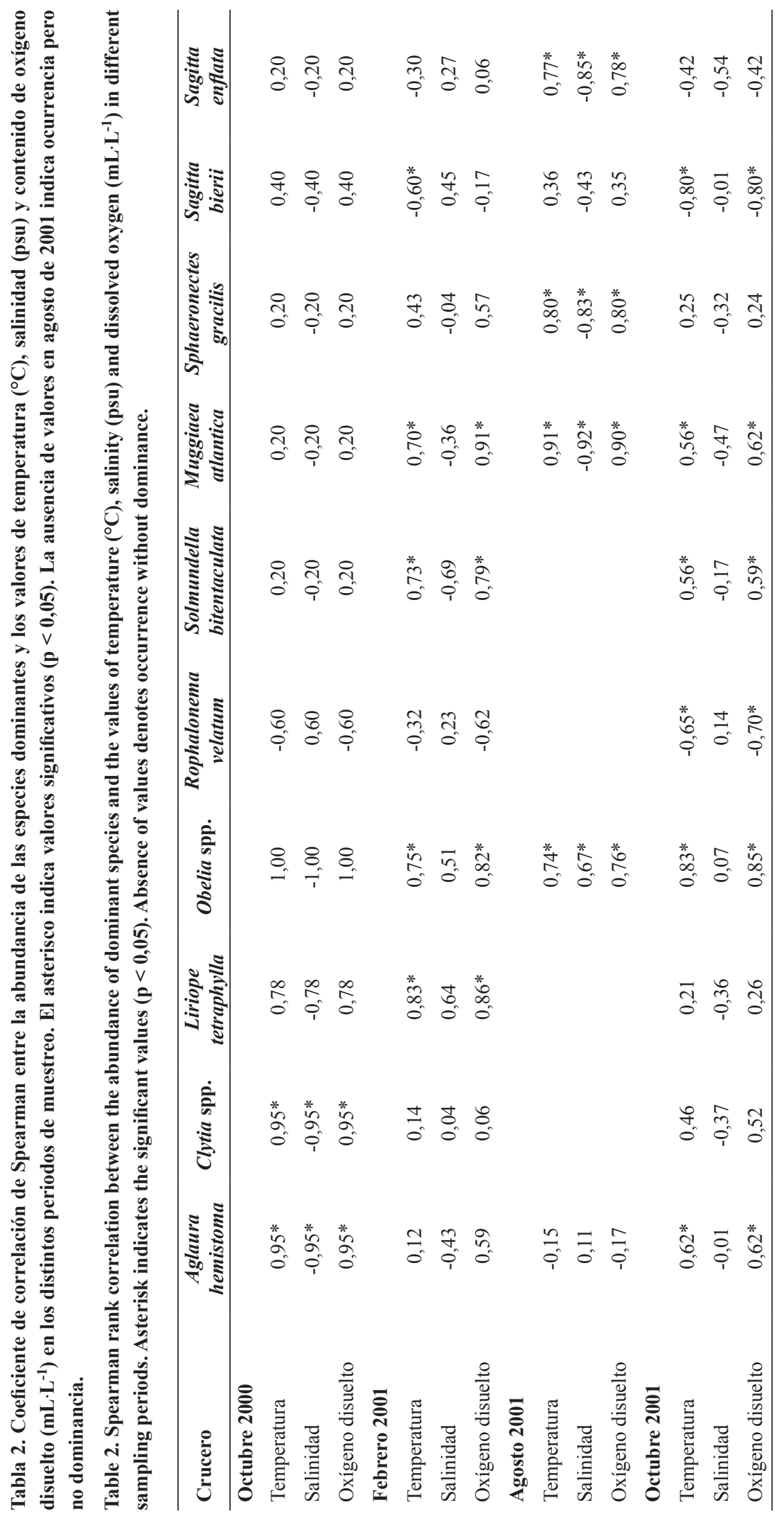




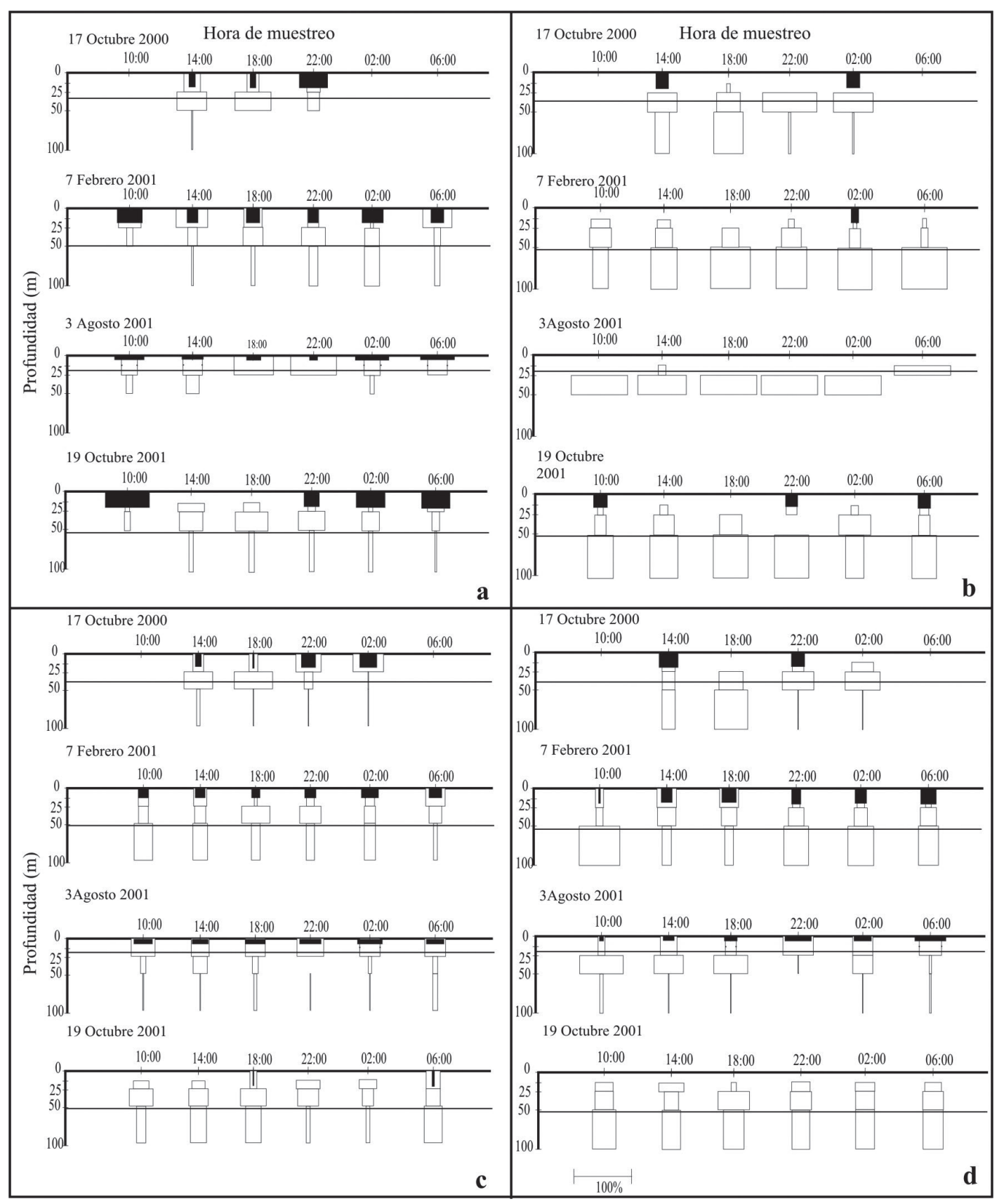

Figura 5. Distribución nictimeral de a) Sphaeronectes gracilis, b) Rophalonema velatum, c) Sagitta enflata y d) Sagitta bierii, en los distintos periodos de muestreo. La barra negra indica el porcentaje de individuos colectados sobre la termoclina y la línea negra, la profundidad media de la Zona de Mínimo Oxígeno.

Figure 5. Diel distribution of a) Sphaeronectes gracilis, b) Rophalonema velatum, c) Sagitta enflata and d) Sagitta bierii, in different sampling periods. The black bar corresponds to individuals collected above the thermocline. The black line indicates the upper limit of Oxygen Minimum Zone. 


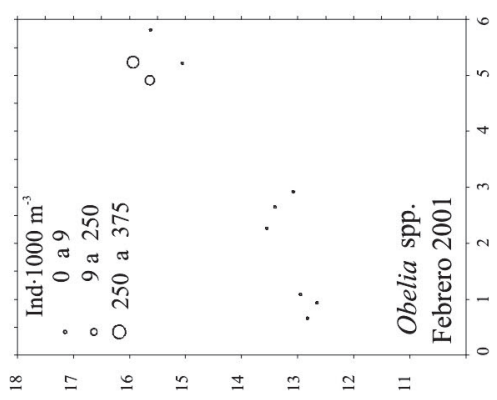

(

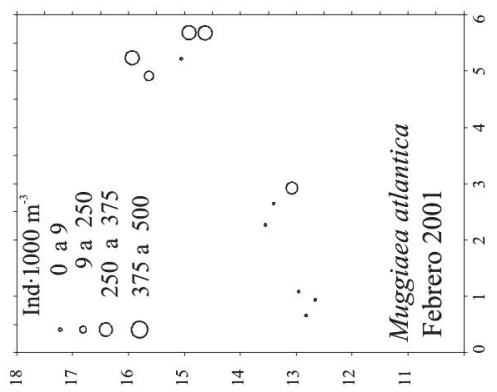

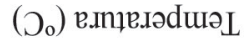

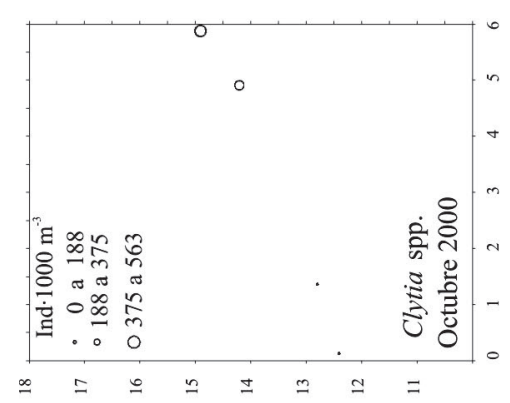

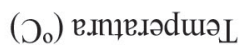

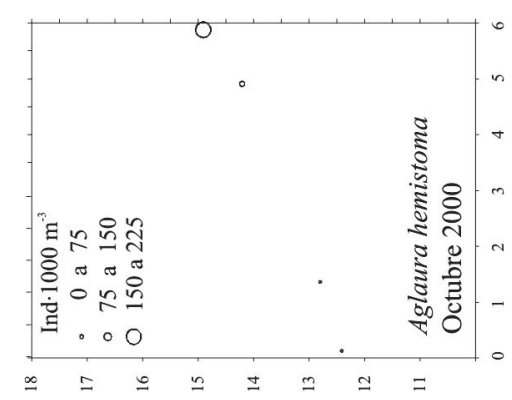

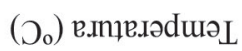



(

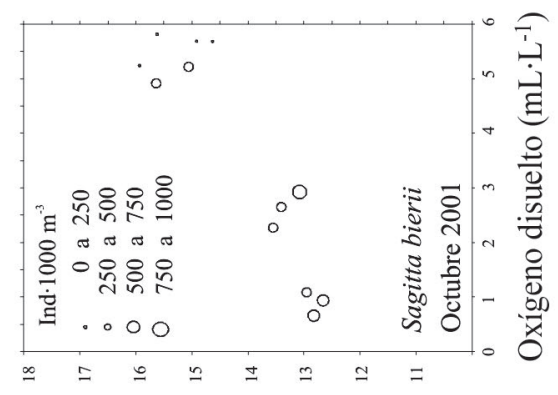

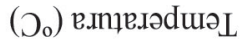

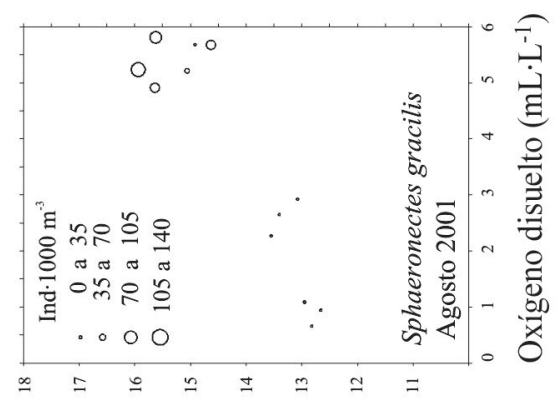

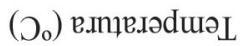

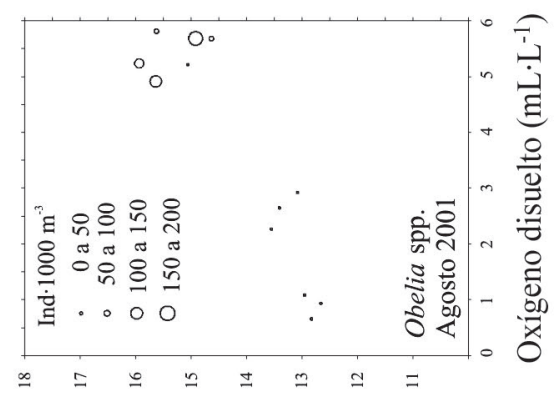

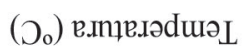


febrero y octubre 2001, alcanzó mayor profundidad (Fig. 5c). En cambio S. bierii siempre se encontró a mayor profundidad que $S$. enflata (Fig. 5d).

La distribución vertical por estrato y horario de muestreo, junto con el análisis de la función discriminante, permitió identificar y separar el comportamiento nictimeral de las especies dominantes en tres grupos (Tabla 3). En octubre de 2000 la mayoría de las especies presentó una migración normal, con mayores densidades diurnas a mayor profundidad ( $25 \mathrm{a}$ $50 \mathrm{~m})$ y nocturnas más someras $(0-25 \mathrm{~m})$, que reflejan pequeños rangos batimétricos de solo alrededor de 15 m diarios, a excepción de $R$. velatum y $S$. bierii que alcanzaron casi los $50 \mathrm{~m}$ (Figs. $5 \mathrm{~b}$ y $5 \mathrm{~d}$ ).

En febrero y agosto, se determinó un predominio de especies no migrantes. En febrero, se detectó una migración inversa en $S$. gracilis y $S$. bierii (Figs. 5 a y 5 d), mientras que en agosto se determinó una migración normal para L. tetraphylla, S. bitentaculata y $S$. bierii. En ambos periodos, las migraciones ocurrieron exclusivamente en los primeros $50 \mathrm{~m}$, con desplazamientos que no superaron los $25 \mathrm{~m}$ diarios (Figs. 4 y 5).

\section{DISCUSIÓN}

Los perfiles verticales de los parámetros oceanográficos mostraron la presencia de una estructura de dos capas en la bahía de Mejillones, una superficial sujeta a fuertes gradientes verticales y otra profunda, más homogénea y estable, de bajo contenido de oxígeno. En la capa superficial las fluctuaciones estacionales fueron causadas por el ciclo anual de temperatura, que causó una intensificación de la termoclina en febrero y un debilitamiento en agosto, y a frecuentes eventos de surgencia que favorecieron el ascenso de la ZMO hacia las capas superficiales, por el ingreso constante del AESS. Lo anterior está de acuerdo con lo señalado previamente para la zona por varios autores, como Escribano (1998), Escribano \& Hidalgo (2000), Escribano et al. (2000), Santibáñez (2003) y Palma \& Apablaza (2004).

En la bahía se identificaron 39 especies gelatinosas, todas las cuales habían sido registradas frente a Antofagasta (Pagès et al., 2001; Palma \& Apablaza, 2004), la mayoría de las cuales son habitantes comunes en aguas subantárticas del SCH (Palma, 1994; Palma \& Rosales, 1995; Ulloa et al., 2000a, 2000b). La abundancia estacional del zooplancton gelatinoso mostró fuertes aumentos en primavera y verano, coincidentes con los incrementos de temperatura, mientras que en invierno se registró una fuerte disminución de abundancia (Tabla 1), que se debería a las menores temperaturas de la columna de agua $\left(13-14^{\circ} \mathrm{C}\right)$ y a la escasa concentración de oxígeno disuelto, ya que solo los primeros $20 \mathrm{~m}$ estuvieron bien oxigenados, ya que bajo esta profundidad, los valores fueron menores de $0,5 \mathrm{~mL} \cdot \mathrm{L}^{-1}$ (Fig. 2). Por otra parte, las mayores densidades de zooplancton observadas en la primavera de 2001 con respecto a la del 2000, serían probablemente resultado del mayor calentamiento $\left(>1^{\circ} \mathrm{C}\right)$ y mayor oxigenación de la columna de agua hasta los $100 \mathrm{~m}$ de profundidad.

Se ha visto que el incremento de temperatura superficial favorece la aparición y desarrollo explosivo

Tabla 3. Clasificación del comportamiento nictimeral de las especies dominantes en los distintos periodos del año.

Table 3. Classification of nycthimeral behavior of dominant species in different periods of the year.

\begin{tabular}{lcccc}
\hline Especie & Octubre 2000 & Febrero 2001 & Agosto 2001 & Octubre 2001 \\
\hline Obelia spp. & 2 & 3 & 3 & 1 \\
R. velatum & 1 & 3 & 3 & 3 \\
S. bitentaculata & 1 & 3 & 1 & 1 \\
M. atlantica & 1 & 3 & 3 & 1 \\
S. gracilis & 1 & 2 & 3 & 1 \\
S. bierii & 1 & 2 & 1 & 3 \\
S. enflata & 1 & 3 & 3 & 1 \\
L. tetraphylla & - & 3 & 1 & 1 \\
\hline
\end{tabular}

1 = Migración normal, 2 = Migración inversa, 3 = No migrante, - ausente en el periodo. 
de algunas especies oportunistas, que pueden formar densas agregaciones costeras particularmente durante el periodo estival, causando cambios importantes en la estructura comunitaria (Milne \& Corey, 1986; Palma, 1994; Palma \& Rosales, 1995). Sin embargo, en la zona de Mejillones, los máximos de plancton gelatinoso se determinaron en primavera cuando ocurren eventos de surgencia que favorecen el desarrollo de altas tasas de producción primaria (Marín et al., 1993), esenciales para el mantenimiento de poblaciones de herbívoros, como Calanus chilensis (Escribano \& McLaren, 1999). Cabe señalar que los copépodos constituyen las principales presas de los organismos gelatinosos (Purcell, 1997).

Estudios previos realizados en copépodos (Escribano \& Rodríguez, 1994; Escribano \& Hidalgo, 2000), muestran que las altas tasas de reproducción y crecimiento representan una estrategia para mantener las poblaciones al interior de la bahía, dado que un porcentaje importante de organismos $(\sim 30 \%)$ son advectados mar afuera en épocas de surgencia. La presencia continua de eudoxias de $M$. atlantica y $S$. gracilis registrada en este estudio y reportada también por Palma \& Apablaza (2004), indica que sus periodos reproductivos son más amplios que en la zona central (Palma, 1977; Palma \& Rosales, 1995). Por lo tanto, es probable que algunas especies gelatinosas utilicen la misma estrategia que los copépodos.

La mayoría de las especies dominantes presentó un escaso rango de distribución vertical $(\sim 50 \mathrm{~m})$, debido a la proximidad de la ZMO a superficie (Figs. 2, 4 y 5). Esto fue confirmado por el análisis de Spearman, que en algunos periodos del año mostró una correlación positiva $(\mathrm{p}<0,05)$ de la abundancia de varias especies dominantes con la temperatura y el contenido de oxígeno disuelto (Fig. 6). Esto significa que la distribución vertical estuvo más restringida a la capa superficial, más cálida y rica en oxígeno. Por lo tanto, la ZMO constituye una barrera química para la distribución y migración vertical de varias especies, pues cuando la ZMO fue más superficial (octubre 2000 y agosto 2001), el plancton estuvo restringido a los primeros $50 \mathrm{~m}$ (Figs. 4 y 5). Este mismo efecto de la ZMO se ha señalado frente a Mejillones para algunos copépodos (Escribano et al., 2000; Santibáñez, 2003), como también frente a Perú, para copépodos (Oncaea, Paracalanus y Centropages) y poliquetos pelágicos (Judkins, 1979).

Un alto porcentaje de los organismos zooplanctónicos realiza desplazamientos nictimerales inducidos por factores físicos y biológicos, como intensidad de la luz, mezcla vertical, alimentación, depredación y ganancia energética (Enright, 1977). Este comportamiento también se ha demostrado en diversas especies gelatinosas (Palma, 1985; Pagès \& Gili, 1991; Andersen et al., 1992). Sin embargo, es probable que en bahía de Mejillones estos desplazamientos sean atenuados no sólo por la escasa profundidad de la columna de agua, sino también por la barrera química impuesta por la ZMO, ya que sus desplazamientos nictimerales fueron de escasa magnitud $(<50 \mathrm{~m}$ ó $<25 \mathrm{~m})$ e incluso algunas especies no incursionan en la $\mathrm{ZMO}$, como ocurrió con Obelia spp. (Fig. 4a). Esto fue más notorio aun en invierno, cuando se registró un ascenso de la ZMO hacia la superficie causado por un intenso evento de surgencia, causando una distribución más somera de los zooplancteres (Figs. 4 y 5 ).

Es probable que $R$. velatum sea capaz de soportar bajos niveles de oxígeno disuelto, ya que generalmente se encontró bajo el límite superior de la ZMO (Fig. 5b y 6). Esto se debería a que es una especie eurihalina $(30-35 \mathrm{psu})$, euriterma $\left(5-29^{\circ} \mathrm{C}\right)$ y tolera bajas concentraciones de oxígeno $\left(<0,2 \mathrm{~mL} \cdot \mathrm{L}^{-1}\right)$ (Navas-Pereira \& Vannucci, 1991).

El cambio más significativo fue el alto número de especies no migrantes en febrero y agosto. En ambos periodos, solo cuatro especies realizaron desplazamientos verticales, las que a su vez disminuyeron drásticamente sus rangos de migración vertical en comparación a octubre, debido a la escasa profundidad de la ZMO. Por otra parte, en octubre de 2000 y 2001, la mayoría de las especies realizaron desplazamientos, siendo en este periodo cuando se observaron los mayores desplazamientos nictimerales (Figs. 4 y 5 ).

Finalmente, se puede apreciar que para la mayoría de las especies dominantes, la termoclina no constituye una barrera física frente a los desplazamientos nictimerales, pues todas las especies consideradas, en algún momento de su ciclo diario realizaron desplazamientos a través de ella. En cambio, la ZMO constituye una verdadera barrera a los desplazamientos vertical del zooplancton gelatinoso en la bahía de Mejillones.

\section{AGRADECIMIENTOS}

Los autores agradecen a la Comisión Nacional de Investigación Científica y Tecnológica de Chile 
(CONICYT), el financiamiento otorgado a través del Proyecto FONDECY 1000419, que permitió el desarrollo de esta investigación.

\section{REFERENCIAS}

Andersen, V., J. Sardou \& P. Nival. 1992. The diel migrations and vertical distributions of zooplankton and micronekton in the northwestern Mediterranean Sea. 2. Siphonophores, hydromedusae and pyrosomids. J. Plankton Res., 14(8): 1155-1169.

Enright, J.T. 1977. Diurnal vertical migration: adaptive significance and timing. Part I. Selective advantage: a metabolic model. Limnol. Oceanogr., 22: 873-886.

Escribano, R. 1998. Population dynamics of Calanus chilensis in the Chilean eastern boundary Humboldt Current. Fish. Oceanogr., 7(3/4): 245-251.

Escribano, R. \& P. Hidalgo. 2000. Spatial distribution of copepods in the north of the Humboldt Current region off Chile during coastal upwelling. J. Mar. Biol. Ass. U.K., 80: 283-290.

Escribano, R. \& I.A. McLaren. 1999. Production of Calanus chilensis in the upwelling area of Antofagasta, northern Chile. Mar. Ecol. Progr. Ser., 177: 147-156.

Escribano, R. \& L. Rodríguez. 1994. Life cycle of Calanus chilensis Brodsky in bay of San Jorge, Antofagasta, Chile. En: F. Ferrari \& B. Bradley (eds.). Ecology and morphology of copepods. Kluwer Academic Publications, Boston, pp. 289-294.

Escribano, R. \& L. Rodríguez. 1995. Seasonal size variation and growth of Calanus chilensis Brodsky in northern Chile. Rev. Chil. Hist. Nat., 68: 373-382.

Escribano, R., V. Marín \& C. Irribarren. 2000. Distribution of Euphausia mucronata at the upwelling area of Peninsula Mejillones, Northern Chile: the influence of the oxygen minimum layer. Sci. Mar., 64(1): 69-77.

Judkins, D. 1979. Vertical distribution of zooplankton in relation to the oxygen minimum off Peru. DeepSea Res., 27: 475-487.

Longhurst, A.R. 1976. Vertical migration. En: D.H. Cushing \& J.J. Walsh (eds.). The ecology of the seas. Blackwell, Oxford, pp. 116-137.

Mamayev, O.I. 1975. Temperature-salinity analysis of world ocean waters. Elsevier Scientific Series,
11. Elsevier Scientific Publishing Company, Amsterdam, $374 \mathrm{pp}$.

Marín, V. 1997. A simple biology, stage-structured population model of the spring dynamics of Calanus chilensis at Mejillones del Sur Bay, Chile. Ecol. Model., 105: 65-82.

Marín, V., L. Rodríguez, L. Vallejo, J. Fuenteseca \& E. Oyarce. 1993. Efectos de la surgencia costera sobre la productividad primaria de bahía Mejillones del Sur (Antofagasta, Chile). Rev. Chil. Hist. Nat., 66: 479-491.

Milne, W.R. \& S. Corey. 1986. Distribution patterns of the ctenophores Pleurobrachia pileus and Beroe cucumis in the Bay of Fundy region, Canada. Can. J. Zool., 64(11): 2639-2644.

Navas-Pereira, D. \& M. Vannucci. 1991. The hydromedusae and water masses of the Indian Ocean, Bolm. Inst. Oceanogr., S. Paulo, 39(1): 25-60.

Olivares, G. 2000. Mecanismos de interacción físico-biológica en una zona de surgencia costera, retención de larvas y cierre del ciclo de vida de Euphausia mucronata. Tesis de Magíster en Ciencias Biológicas mención Ecología, Universidad de Chile, Santiago, 50 pp.

Pagès, F. \& J.M. Gili. 1991. Vertical distribution of epipelagic siphonophores at the confluence between Benguela waters and the Angola Current over 48 hours. Hydrobiologia, 216/217: 355-362.

Pagès, F. \& J.M. Gili. 1992. Siphonophores (Cnidaria, Hydrozoa) of the Benguela Current (southeastern Atlantic). Sci. Mar., 56(suppl.1): 65-112.

Pagès, F., H. González, M. Ramón, M. Sobarzo \& J.M. Gili. 2001. Gelatinous zooplankton assemblages associated with water masses in the Humboldt Current System and potential predatory impact by Bassia bassensis (Siphonophora: Calycophorae). Mar. Ecol. Progr. Ser., 210: 13-24.

Palma, S. 1977. Contribución al estudio de los sifonóforos encontrados frente a la costa de Valparaíso. Aspectos ecológicos. Memorias del Segundo Simposio Latinoamericano de Oceanografía Biológica. Cumaná, Venezuela, 2: 119-133.

Palma, S. 1985. Migración nictimeral del macroplancton gelatinoso de la bahía de Villefranche-sur-Mer, Mediterráneo noroccidental. Invest. Pesq., Barcelona, 49(2): 261-274.

Palma, S. 1994. Composición y distribución del macroplancton gelatinoso recolectado frente a la 
costa central de Chile. Rev. Biol. Mar., Valparaíso, 29(1): 23-45.

Palma, S. \& P. Apablaza. 2004. Abundancia estacional y distribución vertical del zooplancton gelatinoso carnívoro en un área de surgencia en el norte del Sistema de la Corriente de Humboldt. Invest. Mar., Valparaíso, 32(1): 49-70.

Palma, S. \& S. Rosales. 1995. Composición, abundancia y distribución estacional del macroplancton de la bahía de Valparaíso. Invest. Mar., Valparaíso, 23: 49-66.

Pearre, P. 1979. Problems of detection and interpretation of vertical migration. J. Plankton Res., 1: 29-44.

Pillar, S., D. Armstrong \& L. Hutchings. 1989. Vertical migration, dispersal and transport of Euphausia lucens in the southern Benguela Current. Mar. Ecol. Progr. Ser., 53: 179-190.

Purcell, J.E. 1997. Pelagic cnidarians and ctenophores as predators: selective predation, feeding rates, and effects on prey populations. Ann. Inst. Océanogr. Paris, 73: 125-137.

Recibido: 19 enero 2005; Aceptado 20 octubre 2006
Santibáñez, P. 2003. Distribución y abundancia de zooplancton quitinoso en relación a condiciones hidrográficas estacionales frente a la bahía de Mejillones $\left(23^{\circ} \mathrm{S}\right)$, Chile. Tesis de Biología Marina, Escuela de Biología Marina, Universidad Austral de Chile, Valdivia, 56 pp.

Ulloa, R., S. Palma \& N. Silva. 2000a. Bathymetric distribution of chaetognaths and their association with water masses off the coast of Valparaiso, Chile. Deep-Sea Res., 47(11): 2009-2027.

Ulloa, R., S. Palma, L. Linacre \& N. Silva. 2000b. Seasonal changes in the bathymetric distribution of siphonophores, chaetognaths and euphausiids associated to water masses off Valparaíso, Chile (Southeast Pacific). En: J. Farber (ed.). Oceanography in the Eastern Pacific. CICESE, Ensenada, 1: 72-83.

Valdés, J. \& L. Ortlieb. 2001. Paleoxidación subsuperficial de la columna de agua en la bahía de Mejillones del Sur $\left(23^{\circ} \mathrm{S}\right)$ : indicadores geoquímicos en testigos de sedimentos marinos. Invest. Mar., Valparaíso, 29(1): 25-35. 